FSU-SCRI-97C-107

September 1997

\title{
The Schrödinger functional running coupling with staggered fermions and its application to many flavor $\mathrm{QCD}^{* \dagger}$
}

\author{
Urs M. Heller ${ }^{\mathrm{a}}$ \\ aSCRI, The Florida State University, Tallahassee, FL 32306-4130, USA
}

\begin{abstract}
We discuss the Schrödinger functional in lattice QCD with staggered fermions and relate it, in the classical continuum limit, to the Schrödinger functional regularized with Wilson fermions. We compute the strong coupling constant defined via the Schrödinger functional with staggered fermions at one loop and show that it agrees with the continuum running coupling constant in the Schrödinger functional formalism. We compute this running coupling in the "weak coupling phase" of many flavor QCD numerically at several values of the bare coupling and for several system sizes from $L / a=4$ to 12 . The results indicate that the $\beta$-function for 16 flavors has the opposite sign than for few flavor QCD, in agreement with a recent claim, and with the perturbative prediction.
\end{abstract}

\section{THE SCHRÖDINGER FUNCTIONAL WITH STAGGERED FERMIONS}

The Schrödinger functional describes the evolution of a state at (Euclidean) time $t=0$ to another state at time $t=T$. Using the transfer matrix it can be written as a path integral with fixed boundary conditions at time $t=0$ and $T$. For staggered fermions all degrees of freedom can be fixed at both boundaries [1]. The Schrödinger functional can thus be represented as the path integral

$$
\begin{array}{r}
\mathcal{Z}\left[W, \zeta, \bar{\zeta} ; W^{\prime}, \zeta^{\prime}, \bar{\zeta}^{\prime}\right]=\int[D U] \\
\int \prod_{\vec{x}} \prod_{x_{4}=1}^{T-1}\left[d \bar{\chi}\left(\vec{x}, x_{4}\right) d \chi\left(\vec{x}, x_{4}\right)\right] \mathrm{e}^{-S_{G}-S_{S F}}
\end{array}
$$

Here $W$ and $W^{\prime}$ represent the boundary values of the gauge fields and $\zeta, \bar{\zeta}, \zeta^{\prime}$ and $\bar{\zeta}^{\prime}$ those of the fermion fields. The pure gauge action and measure are as in 22. The fermionic part of the action is the usual staggered action with $\chi$ and $\bar{\chi}$ set to zero for $x_{4}<0$ and $x_{4}>T$, and to their boundary values at the boundaries [1, 3].

Note that for staggered fermions the total number of time-slices has to be even. Therefore the time extent $T$ has to be odd. In the spatial direction we take the lattice to be of size

${ }^{*}$ To appear in the proceedings of Lattice '97, Edinburgh, Scotland, July 22-26, 1997.

${ }^{\dagger}$ Work supported by DOE grants DE-FG05-85ER250000 and DE-FG05-96ER40979.
$L$ (even!) and impose the generalized periodic boundary conditions [4] $\chi(x+L \hat{k})=\mathrm{e}^{i \theta_{k}} \chi(x)$, $\bar{\chi}(x+L \hat{k})=\bar{\chi}(x) \mathrm{e}^{-i \theta_{k}}$.

It can be shown [3] that for massless staggered quarks the classical continuum limit of the Schrödinger functional agrees with that from Wilson fermions [5].

\section{THE SCHRÖDINGER COUPLING AT ONE LOOP}

The "Schrödinger functional coupling constant" is defined from the response to a constant chromoelectric background field introduced via the boundary fields. We use those given in [2, 4], which depend on a parameter, $\eta$, and define

$$
\begin{aligned}
\frac{k}{\bar{g}^{2}} & =-\left.\frac{\partial}{\partial \eta} \log \mathcal{Z}\right|_{\eta=0}, \\
k & =12\left(\frac{L}{a}\right)^{2}\left[\sin \left(\frac{2 \pi a^{2}}{3 L T}\right)+\sin \left(\frac{\pi a^{2}}{3 L T}\right)\right],
\end{aligned}
$$

with $T=L$ such that $\bar{g}^{2}$ depends only on one scale, $\bar{g}^{2}=\bar{g}^{2}(L)$. The normalization $k$ has been chosen such that $\bar{g}$ equals the bare coupling at tree-level without any cutoff effects.

The one-loop contribution from the staggered fermions to the coupling constant, $\bar{g}^{2}=g_{0}^{2}+$ $\left(p_{1,0}+n_{f} p_{1,1}\right) g_{0}^{4}+\mathcal{O}\left(g_{0}^{6}\right)$, comes from the derivative of the fermion fluctuation determinant

$p_{1,1}=\left.\frac{1}{k n_{f}} \frac{\partial}{\partial \eta} \log \operatorname{det} M\right|_{\eta=0}$. 
Table 1

The first two 'non-log' terms in the expansion eq. (4) of $p_{1,1} \cdot r_{1}^{(a v)}$ is the average of the choices $T=L \pm a$.

\begin{tabular}{|c|l|l|}
\hline$\theta$ & \multicolumn{1}{|c|}{$r_{0}$} & \multicolumn{1}{c|}{$r_{1}^{(a v)}$} \\
\hline 0 & $-0.004416(1)$ & $0.00947(1)$ \\
$\pi / 5$ & $-0.00579695(2)$ & $0.009477(5)$ \\
1.0 & $-0.0068642(1)$ & $0.00947(1)$ \\
\hline
\end{tabular}

Here $n_{f}=4$ for one flavor of staggered fermions, since they correspond to four flavors of continuum fermions. The fermion boundary fields are set to zero, and $M$ is the fermion matrix.

To be precise, the coupling is defined in 2, 4 as eq. (2) with $T=L$ so that it depends on a single scale, $L$. Unfortunately this is not possible for staggered fermions, since, as we have seen, $L / a$ must be even but $T / a$ must be odd. Instead, we average the couplings obtained with $T=L+a$ and $T=L-a$ to avoid an additional $\mathcal{O}(a)$ effect.

We have evaluated [3] $p_{1,1}^{( \pm)}$of eq. (3) for $L / a$ ranging from 4 to 64 , in steps of 2 . Here the superscript \pm stands for the choices $T=L \pm a$. One can then extract the first few coefficients of the expected asymptotic form

$$
\begin{aligned}
p_{1,1}(L / a) & =r_{0}+s_{0} \log (L / a) \\
& +\left(r_{1}+s_{1} \log (L / a)\right)(a / L)+\cdots .
\end{aligned}
$$

$s_{0}$ should just be $2 b_{0,1}=-1 /\left(12 \pi^{2}\right)$, the fermionic contribution, per flavor, to the $\beta$ -

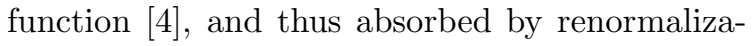
tion. We indeed found this result. $s_{1}$ was found to be compatible with zero. $r_{0,1}$ are listed in Table 11. $r_{0}$ obtained from the choices $T=L \pm a$ always agreed to the accuracy given.

$r_{0}$ of eq. (田) is the finite part of the fermionic contribution to the one-loop relation between bare lattice and running Schrödinger functional coupling. When converted to a relation between the $\overline{\mathrm{MS}}$ and the Schrödinger functional coupling, we found [3] good agreement with the results of Sint and Sommer from Wilson fermions [4]. This confirms that our Schrödinger functional for massless staggered fermions is a correct regularization of the continuum Schrödinger functional with massless fermions.

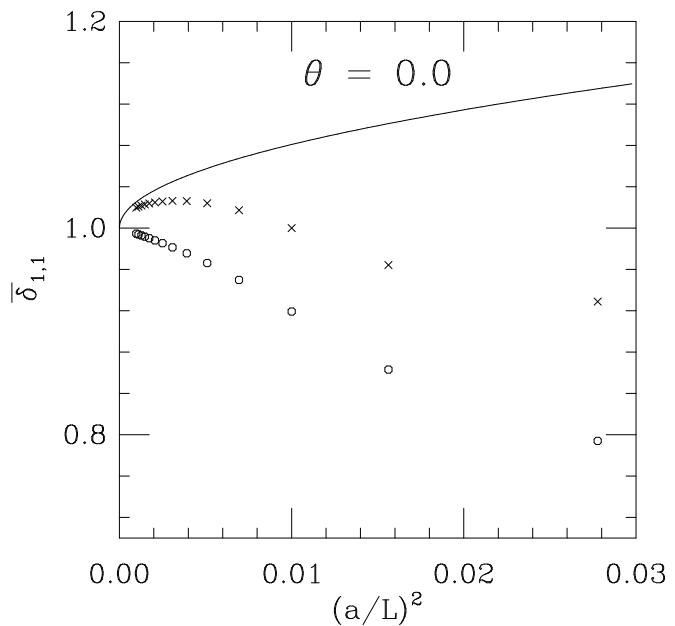

Figure 1. The ratio $\bar{\delta}_{1,1}(a / L)$ (5), for $\theta=0$. Crosses show the result before cancellation of the $\mathcal{O}(a)$ part by the pure gauge boundary counterterm, and octagons the result after the cancellation. The line shows the $\mathcal{O}(a)$ part of the lattice artefact that is cancelled by the counterterm.

\subsection{Lattice artefacts}

The vanishing of $s_{1}$ indicates the absence of bulk $\mathcal{O}(a)$ artefacts. The non-vanishing of $r_{1}^{(a v)}$ reflects the presence of boundary $\mathcal{O}(a)$ effects. These can be absorbed into a pure gauge boundary counterterm [3].

But higher order lattice artefacts, both from the bulk and the boundary, remain. We can study them for the fermionic contribution to the step scaling function [2, 14 with scale factor 2 . We compare the fermion contribution on the lattice (per continuum flavor) with its continuum limit:

$\bar{\delta}_{1,1}(a / L)=\frac{p_{1,1}^{(a v)}(2 L / a)-p_{1,1}^{(a v)}(L / a)}{2 b_{0,1} \log 2}$.

$\bar{\delta}_{1,1}(a / L)$ is shown in Figs. 1 1 and 2 for $\theta=0$ and $\pi / 5$, respectively. Its deviation from 1 at finite $a / L$ is a lattice artefact. As can be seen from the two cases, the higher order lattice artefacts can depend very sensitively on the observable considered, here the step $\beta$-function for different values of the spatial boundary conditions. 


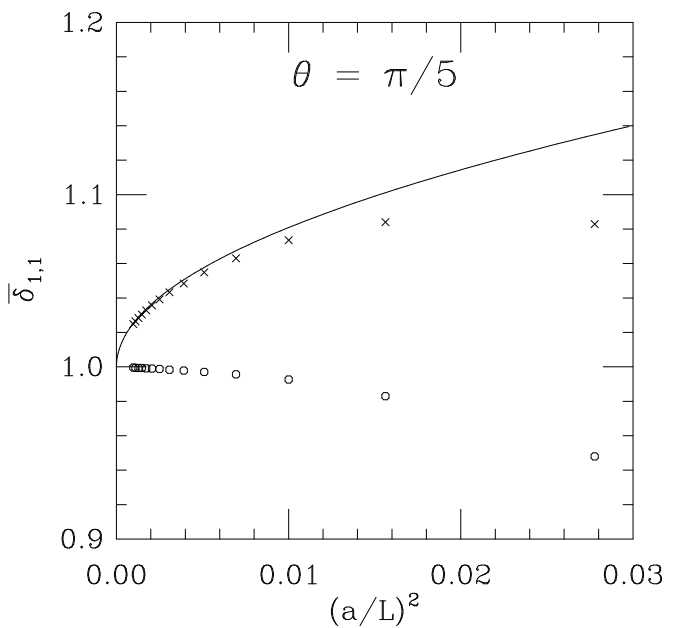

Figure 2. Same as Fig. 1, but for $\theta=\pi / 5$.

\section{APPLICATION TO 16 FLAVOR QCD}

For $n_{f}$ between 9 and 16 the perturbative twoloop $\beta$-function has a second zero, that for $n_{f}=$ 16 occurs at a coupling, $g^{2} \approx 0.5$, where the perturbative prediction might be trusted. In a recent study of $n_{f}=16$ flavor lattice QCD with staggered fermions we found a bulk first order transition from a confined, chirally broken phase at strong coupling to a phase that appeared deconfined and chirally symmetric [6]. Measurement of masses from correlation functions with pion, rho and nucleon quantum numbers indicated that the $\beta$-function in this weak coupling phase might have the opposite sign than few flavor QCD, as perturbation theory predicts. However, in a deconfined phase it is not so clear, what is meant by "hadron masses" and how to relate their behavior to the $\beta$-function, which is defined as the change of the coupling under a change of scale.

The Schrödinger functional formalism gives a direct definition of a running coupling as a function of scale, given by the size of the system. We have measured this coupling at several values of the bare coupling in the "weak coupling phase", and for several system sizes, in numerical simulations. These simulations have been done at zero quark mass, which is possible with the fixed boundary conditions in the Schrödinger
Table 2

The Schrödinger functional coupling, $\bar{g}^{2}(L)$, for $n_{f}=16$, with $1 / \bar{g}^{2}$ averaged over the choices $T=$ $L \pm 1$, for various bare couplings and system sizes.

\begin{tabular}{|c|l|l|l|l|}
\hline$\beta$ & \multicolumn{1}{|c|}{$L=4$} & \multicolumn{1}{|c|}{$L=6$} & \multicolumn{1}{|c|}{$L=8$} & \multicolumn{1}{|c|}{$L=12$} \\
\hline 4.5 & $5.31(15)$ & $3.50(16)$ & $3.22(14)$ & $2.87(12)$ \\
4.6 & $4.41(16)$ & $2.97(5)$ & $2.77(9)$ & \\
4.7 & $3.92(11)$ & $2.78(4)$ & & \\
4.8 & $3.30(6)$ & $2.67(3)$ & $2.37(3)$ & \\
4.9 & $3.07(7)$ & $2.42(3)$ & & \\
5.0 & $2.91(7)$ & $2.28(3)$ & & \\
\hline
\end{tabular}

functional. This avoids having to deal with the anomalous dimension of the quark mass. The results, with $1 / \bar{g}^{2}$ - the observable in the simulations (see (2D) - averaged over the choices $T=L \pm 1$, are listed in Table 2 .

We see, from Table 2, that at fixed bare coupling, $\beta$, the running coupling decreases with increasing system size. This is just the opposite behavior than that exhibited by few flavor QCD: the $\beta$-function of the renormalized coupling has the opposite sign. On the other hand, demanding that $\bar{g}^{2}\left(L / 2, \beta^{\prime}\right)=\bar{g}^{2}(L, \beta)$, i.e. that the lattice spacing at bare coupling $\beta^{\prime}$ is a factor of two larger than at $\beta$, we see that for $n_{f}=16 \beta^{\prime}$ is larger than $\beta$, again, opposite the behavior of few flavor QCD. Hence the $\beta$-function of the bare coupling has the opposite sign. These findings are in agreement with the prediction from perturbation theory, and confirm the results of [6].

\section{REFERENCES}

1. S. Miyazaki and Y. Kikukawa, hep-lat/9409011.

2. M. Lüscher, R. Sommer, P. Weisz and U. Wolff, Nucl. Phys. B413 (1994) 481.

3. Urs M. Heller, hep-lat/9705012, to appear in Nucl. Phys. B.

4. S. Sint and R. Sommer, Nucl. Phys. B465 (1996) 71.

5. S. Sint, Nucl. Phys. B421 (1994) 135; Nucl. Phys. B451 (1995) 416.

6. P.H. Damgaard, U.M. Heller, A. Krasnitz and P. Oleson, Phys. Lett. B400 (1997) 169. 